\title{
EL PROBLEMA DEL TIEMPO EN LA ONTOLOGÍA ORIENTADA A LOS OBJETOS: UN ENCUENTRO ENTRE DELEUZE Y HARMAN
}

\author{
THE PROBLEM OF TIME IN THE OBJECT-ORIENTED \\ ONTOLOGY: AN ENCOUNTER BETWEEN DELEUZE \\ AND HARMAN
}

\author{
Prof. Dr. Eduardo Alberto León ${ }^{1}$ \\ FLACSO, Ecuador
}

\begin{abstract}
Resumen: Este artículo pretende contribuir y desarrollar a una de las principales críticas a la ontología orientada a los objetos en su formulación actual propuesta por Graham Harman en su concepto de tiempo que, tras una inspección más cercana, hace que la teoría general sea inconsistente. Si bien este es el caso, la noción de tiempo de Harman puede modificarse de manera que deje intacto el marco de la ontología orientada a objetos. Aunque, esta teoría ya se puede encontrar en Lógica del Sentido (2011) de Gilles Deleuze. Gran parte de esta última teoría ya se presupone implícitamente en la ontología de Harman, y en este sentido, veremos que la modificación propuesta aborda con éxito las críticas más destacadas que se han expresado a la noción de tiempo de Harman.
\end{abstract}

Descriptores: Ontología $\cdot$ Tiempo $\cdot$ Realidad $\cdot$ Objetos sensuales $\cdot$ Virtualidad

\begin{abstract}
This paper aims to contribute and to develop one of the main criticisms of the object-oriented ontology in its current formulation by Graham Harman, which is a notion of time that, upon closer inspection, reduces the general theory to an inconsistent view. While this is the case, Harman's notion of time can be adjusted in a way that leaves the framework of the object-oriented ontology intact. Although, this theory can already be found in Gilles Deleuze's Logic of Sense (2011), much of this theory is already implicitly presupposed in Harman's ontology, and in this sense, we will see that the proposed modification effectively addresses the most prominent criticisms that have been articulated on Harman's notion of time.
\end{abstract}

Keywords: Ontology $\cdot$ Time $\cdot$ Reality $\cdot$ Sensual objects $\cdot$ Virtuality

Enviado: 11/08/2021. Aceptado: 11/11/2021

\section{INTRODUCCIÓN}

La ontología orientada a los objetos que es parte del realismo especulativo ha tenido un impacto particularmente aceptable al revivir el interés en la metafísica especulativa y la ontología a lo largo de los bordes juveniles de la filosofía académica y principalmente de la filosofía continental. No obstante, una de las críticas más

\footnotetext{
1 Facultad Latinoamericana de Ciencias Sociales, Quito. Departamento de Sociología. E-mail: alberto3026@yahoo.es
} 
destacadas a la filosofía de Harman tiene que ver con su noción del tiempo, puesto que esta es vista como un fenómeno superficial que no afecta a los objetos reales haciendo que toda su ontología sea inconsistente. En este aspecto, como veremos más adelante, tanto para Peter Gratton como Peter Wolfendale, quienes sustentan que una noción defectuosa del tiempo es el aspecto más problemático de la ontología orientada a los objetos. Mi argumento en este artículo será que la actual teoría del tiempo de Harman es, en efecto, defectuosa, pero se puede modificar para convertirla en una teoría más consistente y coherente, conservando el marco general de su ontología.

En este sentido, en primer lugar, se trazarán algunas características importantes de la ontología orientada a los objetos de Harman. Para luego analizar la teoría del tiempo de Harman y sus respectivas críticas por parte de Wolfendale y Gratton. Afirmo que, a la luz de estas críticas, la noción de tiempo de Harman debería ser modificada en una doble noción de tiempo que fue propuesta originalmente por Deleuze en Lógica del Sentido (2011). A partir de esto, se mostrará la sorprendente similitud entre el modelo de realidad que anima las páginas de Lógica del sentido y la de la ontología orientada a los objetos, para luego explicar que esta teoría puede incorporarse a la ontología de Harman con relativa facilidad, ya que esta modificación constituye una respuesta adecuada a las críticas de Wolfendale y Gratton. Finalmente, sostengo como la sorprendente compatibilidad de una teoría deleuziana del tiempo con el modelo cuádruple de entidades de Harman arroja nueva luz sobre la apreciada distancia entre las filosofías de Deleuze y Harman.

\section{LA ONTOLOGÍA ORIENTADA A LOS OBJETOS EL CASO DE HARMAN}

El principio central de la ontología orientada a los objetos es que los objetos no pueden reducirse a sus componentes, ni a sus relaciones con las entidades circundantes, ni tampoco a una combinación de ambos. Los objetos reales se relacionan con "entidades humanas y no humanas, las cuales entran en una interacción simétrica por igual" (Jiménez, 2020, p. 153), de modo que los objetos reales nunca están completamente presentes en la suma de sus partes y sus efectos sobre los demás. Esta es una tesis ontológica más que epistemológica. Esto no quiere decir que el estudio de los componentes o efectos de los objetos nunca produzcan conocimiento. Al contrario, significa que los objetos de ningún modo pueden ser nada más que esos componentes y efectos. También es importante destacar que la ontología orientada a los objetos utiliza el término objeto en un sentido muy general, es decir, "los objetos se presentan con independencia de la conciencia del sujeto. Los objetos no necesitan de la conciencia del sujeto para existir, esto demuestra una extensiva crítica a los principios de la filosofía moderna" (Jiménez, 2020, p. 151). 
Las rocas, las bicicletas, los productos químicos, los colectivos y los edificios son objetos, pero también lo son las granizadas, las personas, las canciones, los ríos, las bacterias, las ciudades, los festivales y los matrimonios. La afirmación es que todos estos podrían contar como objetos reales en la medida en que ninguno de ellos podría reducirse a sus componentes o efectos.

Harman llama "demolición" a los intentos de reducir los objetos a sus partes o a un elemento subyacente. Los ejemplos incluyen filosofías presocráticas que reducen todas las entidades a elementos físicos primordiales, teorías cientificistas que reducen la realidad a partículas subatómicas y filosofías continentales que sostienen que las entidades individuales son concreciones momentáneas de un algo disforme e indeterminado que se esconde detrás de la realidad ordinaria. Harman atribuye esta tesis tanto a "Jean-Luc Nancy como a Emmanuel Levinas" (Harman, 2016, p. 5). La principal razón para rechazar la demolición es que no se puede explicar la emergencia, ya que los objetos tienen propiedades que no se encuentran en los elementos de los que se originan "como productos derivados" (Harman, 2016, p. 6). Un ser humano, por ejemplo, tiene numerosas propiedades que no existirían si sus partes físicas constituyentes estuvieran dispersas a través del universo. Los objetos también son relativamente independientes de sus partes, ya que pueden ganar y perder partes sin dejar de ser ellos mismos. Un colegio, por ejemplo, puede ganar y perder estudiantes, edificios y materias sin dejar de ser ese colegio específico. Por tanto, la ontología orientada a los objetos, se niega a combinar la producción con la reducibilidad. Es indiscutible el asunto de que los objetos sean producidos por otras entidades, pero no se sigue que no sean nada más que estas entidades. A modo de analogía, pensemos en cómo el simple hecho de que las baterías puedan generar electricidad no implica el absurdo de que la electricidad de alguna manera no sea más que baterías.

La reducción de objetos a la forma en que afectan a otras entidades se llama "sepultamiento" (Harman, 2016, p. 7). El problema con el sepultamiento es que no puede explicar el cambio, ya que "comparten la idea de que la existencia de una cosa consiste en relación con otras cosas" (Harman, 2016, p. 8). Si todas las entidades no fueran más que su aprehensión actual por parte de los demás, toda la realidad estaría encerrada en una estasis permanente. Para ayudar a comprender esta idea, tomemos el ejemplo de veinte mil personas que asisten a un concierto de Soda Stereo. Una filosofía del sepultamiento diría, en un momento dado, que Soda Stereo no es más que el hecho de que su audiencia lo experimente, además de cómo puede o no afectar simultáneamente a una multitud de otras entidades. Pero si ese es el caso, sería imposible para Soda Stereo pasar de una canción a la siguiente, ya que su ser estaría completamente exhausto por la experiencia real de la audiencia de su interpretación de la canción actual. Por otra parte, una posición de intemperancia 
implicaría el absurdo de que alguien que llega tarde al concierto nunca pueda empezar a mirar a Soda Stereo, porque solo existirían experiencias de Soda Stereo. La última persona en llegar solo podría comenzar a percibir las experiencias de otras personas y, por supuesto, este también sería el caso de esas personas. Habría más de veinte mil personas que solo perciben las experiencias de otros, sin que nunca haya una entidad que realmente experimenten. Esto es claramente una tontería. Si las cosas pueden verse afectadas, debe haber cosas y no solo más afectos. Por tanto, Harman concluye que los objetos siempre deben ser más que "la suma de cómo están registrados actualmente por otras entidades" (Harman, 2016, p. 23).

Tan pronto como se establece la irreductibilidad de los objetos, podemos deducir que deben tener una estructura ontológica cuádruple. Primero, si un objeto es "irreductible a sus propias piezas, e igualmente irreductible a sus relaciones externas con otras cosas" (Harman, 2016, p. 43), entonces debe ser un objeto real que constituye un exceso ontológico por encima de sus partes y compromisos. En segundo lugar, no puede darse el caso de que lo que otras entidades experimentan, registran o aprehenden sea alguna vez un objeto real, puesto que eso sobrecargaría el objeto en su interior relacionándose con los demás. Lo que se manifiesta en las relaciones de un objeto con otros es en cambio un objeto sensual ${ }^{2}$, que es una traducción de un objeto real donde se "caracteriza todo el ámbito relacional de los objetos, la manera como ellos se relacionan entre sí de distintas e infinitas maneras" (Ramírez, 2017, p. 15) en la experiencia de alguna otra entidad. Al percibir una montaña, por ejemplo, obviamente no es el caso que la montaña misma sea literalmente presente en mi conciencia. Simplemente percibo una manifestación derivada o traducida de la montaña real, y este última se retira detrás de la superficie sensual con la que me enfrento.

Posteriormente, los objetos sensuales no pueden ser simples detalles. Si los objetos solo encuentran la superficie sensual de otros objetos, entonces el hecho de que diferentes encuentros tengan distintos efectos en diferentes objetos exige que los objetos sensuales tengan cualidades específicas. En términos de Harman, los objetos sensuales deben "estar incrustados con cualidades sensuales" (Harman, 2016, p. 43). En este punto, un estudioso de Hume, podría decir que la noción de cualidades sensuales hace que los objetos sensuales sean superfluos. El contenido visible de la experiencia serían simplemente cualidades "como rojo, redondo o dulce" (Harman, 2016, p. 22) y la entidad perceptiva las generalizaría en algo como manzana por la fuerza de la experiencia. La ontología orientada a los objetos, sin embargo, sigue

\footnotetext{
2 "La estructura de los fenómenos conscientes se forja a partir de cualidades eidéticas, o experimentalmente interpretativas, intuidas intelectualmente. Objeto real / Cualidades sensuales: Al igual que en el análisis de herramientas, un objeto retirado se traduce en aprehensión sensual a través de una "superficie" a la que accede el pensamiento y / o la acción" (www.hisour.com).
} 
a Edmund Husserl al sostener que los objetos sensuales se "dan inicialmente en lugar de sintetizarse habitualmente" (Harman, 2016, p. 48). Si doy un paseo por una ciudad que tiene edificios con arquitectura brutalista, ${ }^{3}$ por ejemplo, diferentes colores y características se deslizan dentro y fuera de la vista mientras rodeo la estructura (Casado, 2019). Debido a las variaciones en el estado de ánimo, la iluminación y el fondo, pueden parecer un trozo de hormigón intimidante y grotesco en un momento, mientras que, en otro momento me sorprende como un monumento inspirador al ingenio humano. La ontología orientada a los objetos argumenta que esto legitima la existencia de un edificio que se da en la práctica, pero no está constituido por la experiencia. El juego cambiante de cualidades indica que el edificio es rígido con respecto a cómo se percibe y se usa, de modo que el mismo edificio persiste en las múltiples formas en que se encuentra.

Por último, no puede darse el caso de que el aspecto separado de un objeto no comprenda más que un objeto real. En este punto, Harman sigue el argumento de Leibniz de que las mónadas deben ser más que simples unidades, puesto que también necesitan tener cualidades individuales difieren entre sí, es decir, si existirá una razón suficiente de por qué las manifestaciones sensuales de un objeto difieren de las del siguiente (Harman, 2016). De ahí la estructura cuádruple de los objetos. Por un lado, un doble de un objeto real y sus cualidades reales comprende una esencia irreductible que se aleja de cómo el objeto es percibido o registrado por otros. Por otro lado, un doble de un objeto sensual y sus cambiantes cualidades sensuales constituyen la presencia del objeto en otras entidades.

En este sentido, es importante destacar dos características de la ontología orientada a los objetos que son pertinentes a lo que sigue: finitud ontológica y apertura unilateral. Empezando por el primero, no se da el caso de que sólo los humanos estén consignados a un mundo de superficies sensuales que excluyen el contacto directo con otros objetos reales. Todos los objetos se enfrentan a esta finitud ontológica, ya que ninguna entidad entra en contacto directo con otro objeto real:

"Si nuestra percepción de una silla la reduce a una caricatura unidimensional de su ser secreto oculto, lo mismo vale para mi acción práctica de sentarme en ella, puesto que, en nuestra profunda estupidez, tendemos a despreciar la innumerable cantidad de rasgos de la silla que son relevantes para los perros, los mosquitos y los rayos cósmicos. Hay una profundidad de las cosas que no se agota en ninguna actividad humana, sea consciente o inconsciente". (Harman, 2015, p. 48)

\footnotetext{
3 "El brutalismo se asocia, de una manera inmediata y poco meditada, a gigantescas moles de hormigón armado dominando sobre ciudades sombrías. Los resultados estéticos de esta arquitectura fueron demasiado impactantes y bruscos para la época (décadas de los 50 y 60), provocando en la crítica especializada y en profanos sentimientos de aversión e ira" (Casado, 2019, p.5).
} 
El duelo entre los objetos aislados y sus superficies sensuales, así como la traducción distorsionante de las esencias en apariencias manifiestas, no es, por tanto, una desventaja humana trágica, sino un hecho ontológico. Todo lo que cualquier objeto registra son objetos sensuales y sus cualidades. En segundo lugar, la apertura unilateral es el hecho de que, aunque los objetos reales nunca son experimentados directamente por otras entidades, solo los objetos reales experimentan otras entidades (Baraglia, 2014). En la ontología orientada a los objetos, no existe un objeto sensual que experimente algo, ya que los objetos sensuales son simplemente lo que alguien más experimenta. Por tanto, el objeto que experimenta debe ser "siempre un objeto real" (Harman, 2016, p. 10). Cuando veo a mi pareja en una calle muy transitada, por ejemplo, la entidad que la ve es un yo como objeto real y no un yo como objeto sensual, puesto que esto último implicaría el absurdo de que ella es vista por la percepción que otra persona tiene de mí. O para dar otro ejemplo: dado que los océanos, los perros y los humanos son todos afectados por la luna de diversas formas, lo que les afecta son las manifestaciones sensuales de la luna, pero las entidades que registran estos afectos son todos objetos reales.

\section{LOS VÍNCULOS CON EL TIEMPO}

Si los objetos cuádruples son los ingredientes más básicos de la realidad, como sostiene la ontología orientada a los objetos, entonces tales objetos no pueden existir en algo que no es en sí mismo un aspecto de otro objeto similar. De ello se desprende que la ontología orientada a los objetos debe dar cuenta de todas las características sobresalientes de la realidad en términos de los "cuatro aspectos de los objetos y los diez posibles vínculos entre ellos seis vínculos entre los cuatro aspectos y cuatro vínculos que refieren cada aspecto respectivo a sí mismo" (Harman, 2017, p. 57). Lo mismo ocurre con el espacio y el tiempo, que según Harman deben explicarse en términos de "la polarización entre los objetos y sus cualidades" (Harman, 2016, p. 34). Ya que es el tiempo lo que nos ocupa aquí, por ahora simplemente notamos de pasada que Harman ${ }^{4}$ define el espacio como la tensión entre un objeto real y sus cualidades sensuales. El hecho de que haya espacio y no una compresión totalmente inmediata de todo se debería a que los objetos reales se retiran detrás de sus manifestaciones sensuales, con lo que literalmente hacen lugar.

Harman define el tiempo como la tensión entre los objetos sensuales y sus cualidades sensuales "el objeto sensual estará siempre presente, con todos sus rasgos" (Baraglia, 2014, p. 28). Se basa en lo que considera nuestra experiencia común del tiempo como "una notable interacción de estabilidad y cambio" (Harman,

\footnotetext{
${ }^{4}$ Mi afirmación no es que la noción de espacio de Harman no sea problemática, sino que la modificación propuesta de su noción de tiempo puede realizarse sin analizar su noción de espacio con todo detalle.
} 
2016, p. 89). Por un lado, la experiencia nos presenta combinaciones cambiantes de olores, sonidos, colores y otras cualidades. Por otro lado se "afirma que los datos de los sentidos proveen una descripción estrecha y arbitraria del campo de la experiencia, es decir, de los objetos y sus relaciones mutuas" (Harman, 2015, p. 32). Esto corresponde a la tensión entre los objetos sensuales y las cualidades con las que están incrustados (Harman, 2016). Si volvemos al ejemplo del edificio brutalista, las diferentes formas en las que el mismo edificio me impacta mientras lo rodeo no ocurrirían estrictamente en el tiempo, porque esta dinámica de cambio y mismidad sería en sí mismo tiempo.

Esta dinámica no se produce en el vacío, porque los objetos sensuales son objetos con los que se relacionan. En este ejemplo, yo soy el objeto real que aprehende el juego temporal entre el edificio y sus diferentes cualidades. Además, no encuentro el edificio en el vacío, sino dentro de uno o varios objetos reales. No obstante, todos estos objetos reales "están en cierto sentido fuera del tiempo" (Harman, 2005, p, 250). Según la ontología orientada a los objetos, el edificio real brutalista se aleja de sus apariencias sensuales, por lo que no puede participar en el juego temporal entre el objeto sensual y las cualidades sensuales que yo percibo. Lo mismo ocurre conmigo como objeto real que observa este juego. Puedo experimentar un fenómeno temporal, pero dado que el tiempo se define estrictamente como la tensión entre los objetos sensuales y sus cualidades, la aprehensión misma no es de alguna manera temporal. Por último, algo similar debe ocurrir con cualquier objeto real que sea el medio o entorno en el que me encuentro con el edificio, aunque este objeto mediador sea el lugar de un fenómeno temporal que por definición no está implicado en ese fenómeno concreto.

Algunas de las principales críticas a la idea de tiempo de Harman son las de Peter Wolfendale y Peter Gratton. Ninguno de los dos autores sostiene que la noción de tiempo de Harman sea la única parte ambigua de su ontología, pero ambos coinciden en que es la más problemática. Según Gratton, “el futuro mismo de la ontología orientada a los objetos como teoría viable depende de si puede o no proporcionar una teoría adecuada del tiempo" (Gratton, 2014, p. 107). Wolfendale es aún más feroz y sostiene que las actuales nociones de tiempo y espacio de Harman son "el aspecto más catastróficamente inepto de su sistema metafísico" (Wolfendale, 2014, p. 188).

Gratton señala que "si Harman quiere dar contenido a los objetos, se corre el riesgo de un idealismo peor que todo lo que critica" (Gratton, 2014, p. 99). No se trataría de un idealismo que afirme una identidad entre el ser y el pensamiento o la experiencia, sino de un idealismo de formas ideales que habitan en un "presente eterno, inmutable e inafectado por las vicisitudes ulteriores de la realidad" (ibíd.). Estas formas ideales serían las esencias de los entes y objetos reales con cualidades 
existentes como contenido. El problema aquí no es que la ontología de Harman deje de calificarse como realismo, ya que simplemente sería realista sobre las formas ideales. El problema es que, si los objetos reales están fuera del tiempo, no pueden llegar a existir, ni cambiar, ni perecer, ya que éstas son formas de cambio, en este aspecto vale la pena preguntarse ¿cómo se produce el cambio en lo real, que perdura y está fuera del juego del tiempo en el nivel sensual? Este punto también lo plantea Wolfendale:

"Las cosas deben cambiar en el ámbito subterráneo de los objetos retirados, aunque sólo experimentemos las ondas que estos cambios producen en el mundo de la superficie brillante. Estos cambios reales y el tiempo que presuponen no están explícitamente explicados por lo que pasa por "tiempo" en el nivel del cambio sensual" (Wolfendale, 2014, p. 199).

Si el cambio real, más que el sensual, no existe, entonces toda la realidad no es más que una representación pasiva de un conjunto inmutable de objetos reales con cualidades inalterables, lo que haría de la ontología orientada a los objetos precisamente el tipo de teoría que pretende superar. Además, hay que tener en cuenta que Harman sostiene explícitamente que cualquier objeto real dado sufrirá a veces un cambio real, ya que pasará por "varios usos al mismo tiempo" (Harman, 2015, p, 97), es decir varios puntos de inflexión en su vida.

Harman, en su libro Immaterialism: Objects and Social Theory (2017), esboza una teoría del cambio simbiótico según la cual algunos de los encuentros de un objeto, pero no todos, alterarán sus cualidades reales. Por analogía, comparemos esto con el hecho de que la mayoría de nuestras interacciones diarias no alteran fundamentalmente nuestra personalidad, pero aún así habrá momentos relativamente raros en los que algo nos afecte profundamente y cambie lo que somos. Según Harman, éste es el modelo de cambio real en los seres humanos, así como en las cebras, las bicicletas, los gobiernos y los movimientos sociales (Harman, 2017). A la luz de la apertura unilateral mencionada anteriormente, esto no es ciertamente excluido por los principios básicos de la ontología orientada a los objetos, pero es obvio que la teoría del tiempo de Harman necesita ser reelaborada si quiere dar razones de como los objetos reales experimentan un cambio real.

En estrecha relación con esto hay otros dos problemas identificados por Wolfendale. En primer lugar, a pesar de restringir el tiempo a la tensión entre un objeto sensual y sus cualidades sensuales, Harman utiliza términos inequívocamente temporales para definir los otros nueve vínculos entre los cuatro aspectos de los objetos. Tomemos como ejemplo a alguien que regresa a un lugar que ha sufrido cambios significativos desde su última visita: digamos que vuelvo al barrio donde crecí, pero todas las casas han sido demolidas y sustituidas por fábricas. Según 
Harman, este cambio en el "régimen de los objetos" (Harman, 2005, p. 251) no es un cambio temporal, sino espacial. Recordemos que el espacio se definió como la tensión entre los objetos reales y sus cualidades sensuales. Para Harman, el cambio que noto se debe a que los colores, los olores, las formas y los sonidos en los que me encuentro inmerso son manifestaciones sensuales de nuevos objetos reales que han sustituido a los que antes existían allí.

En este sentido, Wolfendale señala, con toda razón, que cualquier noción de cambio presupone una "noción de tiempo, por lo que es incoherente" (Wolfendale, 2014, p. 78). Si el tiempo es la tensión entre el objeto sensual y las cualidades sensuales; y si el espacio es, en cambio, la tensión entre un objeto real y esas cualidades sensuales, entonces el cambio simplemente no puede pertenecer al espacio.

Otro ejemplo sería el vínculo de contigüidad entre los objetos sensuales. Los objetos sensuales "no pueden entrar en contacto más que a través del mediador que los experimenta" (Harman, 2016, p. 128). Por utilizar uno de los ejemplos de Harman, un conjunto de agujas y torres sólo constituye una línea de horizonte en la medida en que un observador vincula estos objetos sensuales a una línea de horizonte en su experiencia, no obstante, esto también implica tiempo. Supongamos que estoy subiendo a El Panecillo en la ciudad de Quito o el Pan de Azúcar en Brasil y un número creciente de estructuras aparece a la vista. Se está formando una aproximación entre los objetos sensuales a medida que se añaden más objetos sensuales a mi experiencia de la línea del horizonte. Esto implica que el reino sensual no sólo contiene el juego temporal entre los objetos sensuales y sus cualidades cambiantes, sino también un juego similar de asociaciones variables entre los propios objetos sensuales (Harman, 2016). No obstante, si el tiempo es simplemente la tensión entre un objeto sensual y sus cualidades, esto debería ser imposible. Algo similar ocurre en las descripciones de Harman de los siete enlaces restantes, pero como el problema general debería quedar claro a partir de los dos ejemplos que acabamos de dar, no es necesario discutir el resto con todo detalle.

El segundo problema relacionado tiene que ver con el simple hecho de que se supone que "los objetos reales y sus cualidades reales persisten" (Wolfendale, 2014, p. 198). Gran parte de la ontología de Harman se basa en que los objetos reales y sus cualidades no se disuelven en sus compromisos actuales con otras entidades, ya que tal disolución constituiría un exceso de demolición. Los objetos reales deben, por tanto, persistir más allá de sus superficies sensuales, pero esta afirmación carece de sentido mientras dicha persistencia no sea de algún modo temporal. Como escribe Wolfendale, esto implica una dependencia tácita de un segundo "tipo de tiempo profundo" (ibíd.) del que la ontología orientada a los objetos no da cuenta en su formulación actual. 
Por consiguiente, una versión modificada de la teoría del tiempo de Harman debe cumplir al menos tres criterios. En primer lugar, debe permitir la producción, la alteración y la aniquilación de objetos reales con cualidades reales, y hacerlo de una manera que no implique ni el demoler ni el exceso de sepultamiento. ${ }^{5}$ En segundo lugar, debe permitir que los diez vínculos entre los aspectos del cuádruple objeto sean temporales, puesto que estos vínculos se refieren a lo que ocurre en la realidad. En tercer y último lugar, debe dar cuenta de la persistencia de los objetos, pero sin proyectar un tiempo que exista por encima de los objetos, ya que esto violaría la tesis de que los objetos son los componentes básicos de la realidad.

\section{DeleuZe y las dos estructuras del tiempo}

El argumento aquí no se refiere en absoluto a la filosofía más amplia de Deleuze, sino a la parte de la teoría del tiempo de Deleuze, ya que dicha teoría nos da las herramientas conceptuales necesarias para hacer más coherente la filosofía de Harman. En este sentido, buscamos una noción de tiempo que dé cuenta de la temporalidad de los objetos sensuales y reales, además de la temporalidad de los vínculos entre esos niveles. Esta noción se encuentra en La Lógica del Sentido (2011) de Gilles Deleuze, que teoriza el tiempo de una manera doble utilizando los conceptos Aion y Cronos. Algunos pueden pensar que Deleuze teoriza el tiempo de una manera triple en lugar de una forma doble, dadas sus elaboradas descripciones de tres síntesis temporales (pasiva, trascendental, estática) en Diferencia y Repetición (2006) y en otros lugares de su obra. Sin embargo, estas tres síntesis se refieren a lo que las entidades hacen en el tiempo, no al tiempo propiamente dicho. Esto seguirá sonando impreciso por el momento, pero en la siguiente sección se discutirá el estatus de las síntesis temporales dentro de una teoría del tiempo doble con más detalle.

El trabajo de Deleuze, Lógica del Sentido, no puede entenderse sin captar su profundo contraste con Diferencia y Repetición, aunque el primero se publicó sólo un año después del segundo respectivamente 1969 y 1968. Según la conocida ontología de Diferencia y repetición, nuestra realidad cotidiana de entidades individuales aparentemente discretas y discontinuas es el resultado pasivo de procesos mutuamente continuos pero autodiferenciados que se desarrollan en un "reino virtual" intensivo, un reino que es diferente en especie de la extensa actualidad que perciben nuestros sentidos, es decir:

\footnotetext{
${ }^{5}$ Recuérdese la tesis de Harman de que el cambio real es raro, de manera que los objetos no son arrastrados por sus compromisos.

${ }^{6}$ Deleuze, no se distancia explícitamente de esta ontología del reino virtual actual, ya que el par virtual-actual persiste en numerosos textos posteriores a Diferencia y repetición (206), como ¿Qué es la filosofía? (2013), "Lo actual y lo virtual", anexado a Diálogos (1996), y su última publicación, "La inmanencia: una vida" (2002).
} 
"para algo potencial o virtual, actualizarse siempre es crear líneas divergentes que se corresponden sin semejanza con la multiplicidad virtual. A lo virtual le corresponde la realidad de una tarea por cumplir o un problema por resolver; el problema es el que orienta, condiciona genera las soluciones" (Deleuze, 2006, p. 319).

En términos de Harman, esta sería una posición debilitante, ya que las entidades individuales serían expresiones pasivas de una dimensión preindividual más fundamental. Por otro lado, en el prefacio ${ }^{7}$ de Lógica del Sentido, Deleuze, escribe que todavía corresponde a una teoría de profundidad arcaica y que Lógica del Sentido trata en cambio de una nueva teoría de las "superficies" (Deleuze 2011, pág. 6). De hecho, a lo largo treinta y cuatro "series" más dos Apéndices que componen el libro, no hay una sola mención del reino virtual que anima las páginas de Diferencia y Repetición.

El esquema general de esta nueva teoría de las superficies es inmediatamente claro en las primeras secciones de Lógica del Sentido, y se podrá notarán su proximidad. Si lo podemos decir de este modo, con relación a la ontología orientada a los objetos de Harman. Deleuze postula una ontología en la segunda serie de paradojas basada en un dualismo de "cuerpos o estados de las cosas, y de los efectos incorpóreos o acontecimientos" (Deleuze, 2011, p. 2). Un cuerpo debe entenderse como una "sustancia y causa" (Deleuze, 2011, p. 11) con sus propias “cualidades físicas” (ibíd.), y Deleuze agrega que cada cuerpo "se retira de otros cuerpos. Por el contrario, los eventos incorpóreos son simplemente "«casi-causas»" (Deleuze, 2011, p. 10) que dependen de los cuerpos para sus "causas reales" (ibíd.).

Los acontecimientos también tienen sus propias cualidades, que Deleuze llama "atributos" (2011, p. 11). Es decir, los acontecimientos no "tienen" cualidades, sino que se atribuyen a los cuerpos, o sea: son los atributos mismos cuando son referidos a los cuerpos. Así, el acontecimiento-sentido "verdear" se vuelve un atributo del cuerpo “árbol” (cf. Deleuze, 2011, p. 13). Estos acontecimientos y sus atributos forman el mundo de la experiencia sensual, incluyendo árboles que saludan a Alicia con su follaje verde, luz solar que calienta nuestra piel y batallas que asolan nuestras tierras. Si los cuerpos se retiran unos de otros, esto se debe a que los acontecimientos incorpóreos envuelven a los cuerpos y por lo tanto les impiden hacer contacto directo (cf. Deleuze, 2011, p. 11). En este sentido, los acontecimientos incorpóreos son intransitables. Por ende, la realidad es más profunda que las superficies reveladas de los acontecimientos incorpóreos, pero ya no es la profundidad

${ }^{7}$ Nota para la edición italiana de Lógica del sentido (1976), compilada en Dos regímenes de locos (2008, n.5). Ciertamente, Deleuze destaca su desplazamiento desde una teoría de las profundidades en Diferencia y Repetición, a un examen de las superficies en Lógica del Sentido. 
de un reino virtual unificado y continuo. En cambio, es la profundidad de los propios cuerpos cuando se retiran detrás de sus superficies ${ }^{8}$.

Según la ontología de Lógica del Sentido, los cuerpos pueden producirse y modificarse. Los que estén familiarizados con el libro recordarán que Deleuze argumenta con ímpetu contra las teorías platónicas en las que la realidad sensible es una representación de formas eternas e inmutables. Para evitar una regresión a tal platonismo, los cuerpos deben ser cosas que suceden y cambian. Es por eso que Deleuze a veces también escribe sobre cuerpos reales en términos de acontecimientos, por ejemplo, cuando describe una batalla o una vida humana como un acontecimiento, escrito con una A mayúscula para evitar confundir una entidad con sus manifestaciones superficiales. Para evitar el platonismo, Deleuze emplea dos estrategias mancomunadas: la apología del simulacro como lo que destituye tanto el modelo como la copia ( $1^{\mathrm{a}}$ serie, Apéndice 1); lo ideal o lo incorporal (el acontecimiento-sentido) comprendido solo como efecto, nunca como causa ( $2^{\mathrm{a}}$ serie) (Deleuze, 2011).

Deleuze también es consciente de que, si los cuerpos son producidos y alterados, entonces esto debe involucrar acontecimientos incorpóreos, ya que hay mezclas de cuerpos. Por tanto, los sucesos superficiales deben ser el producto de los cuerpos y al mismo tiempo estar presentes en la producción de los cuerpos. Esto lleva a Deleuze a una doble teoría del tiempo. Por un lado, está el tiempo ${ }^{9}$ de los efectos incorpóreos, el tiempo de lo que aprehenden los cuerpos reales. Por otro lado, está el tiempo de lo que les sucede a estos mismos cuerpos. Como escribe Deleuze:

"el tiempo debe ser captado dos veces, de dos modos complementarios, exclusivos el uno de otro: enteramente como presente vivo en los cuerpos que actúan y padecen, pero enteramente también como instancia infinitamente divisible en pasado-futuro, en los efectos incorporales que resultan de los cuerpos, de sus acciones y de sus pasiones" (Deleuze, 2011, p. 9).

Él llama al tiempo de los cuerpos Cronos y se refiere al tiempo de los efectos incorpóreos como el Aion "Cronos es el presente que sólo existe, y que hace del

\footnotetext{
${ }^{8}$ También encontramos este modelo de realidad en la segunda edición del libro de Deleuze, Proust y los Signos (1995). Publicado originalmente en 1964, Deleuze agregó una segunda parte completa a la versión de 1972. El texto recién agregado describe una teoría de los cuerpos considerados como vasijas cerradas que se comunican sólo indirectamente porque sólo registran los signos de los demás. En un parecido sorprendente con los diez vínculos de Harman entre los cuatro aspectos de los objetos, Deleuze subraya que tal teoría de la realidad implica una estructura galáctica de diez combinaciones entre los diversos aspectos de tales entidades.

${ }^{9}$ La teoría de Deleuze puede muy bien concordar con el modelo de tiempo de la ontología orientada a los objetos propuesta por Timothy Morton. Morton escribe que: "Los objetos no se sitúan en una rígida caja temporal. Están "internamente" fuera de fase con ellos mismos, y esto es lo que produce el tiempo y la posibilidad de que interactúen entre sí." (Morton, 2020, p. 202). Esto implica dos sentidos del tiempo distintos: uno para la existencia de un objeto entre otras entidades, y otro para su existencia en una relación tensa desfasada consigo mismo.
} 
pasado y del futuro sus dos dimensiones dirigidas (...) Aión es el pasado-futuro en una subdivisión infinita del momento abstracto, que se descompone sin cesar en los dos sentidos a la vez, esquivando siempre cualquier presente" (Deleuze, 2011, p. 61). Aion $^{10}$ es el tiempo de los "acontecimientos-efectos" (Deleuze, 2011, p. 105) en la superficie de las entidades, que se refiere al juego variable de los acontecimientos incorpóreos y sus cualidades. A diferencia del presente de los cuerpos reales, que existen, los eventos del Aion "subsisten o insisten" (p. 11). Esto suena a meticulosidad escolástica, pero simplemente significa que el juego de los acontecimientos solo existe en relación con otra cosa, ya que los eventos de la superficie solo suceden en relación con los cuerpos que los aprehenden. Por lo tanto, hay un sentido en el que Aion designa la instancia infinitamente divisible en pasado y en futuro de los puros acontecimientos (Deleuze, 2011). Su experiencia actual de leer este artículo, posiblemente con una copa de vino a su lado y con música de fondo, es una variedad aiónica, -si utilizamos el termino de esta manera- de acontecimientos incorpóreos estrechamente entrelazados.

No obstante, hay un sentido aún más fuerte en el que Aion "se ha liberado de su contenido corporal presente" (Deleuze, 2011, p. 120), de modo que "el pasado y el futuro insisten o subsisten en el tiempo" (p. 119). Los eventos incorpóreos son producidos por los cuerpos recordemos que Deleuze llama a todos los cuerpos causas, es decir "todos los cuerpos son causas, causas unos en relación con lo otros unos para otros" (Deleuze, 2011, p. 9). Dado que los cuerpos nunca se encuentran directamente, el juego cambiante de la experiencia no es más que la transición de eventos incorpóreos que estos cuerpos han producido (pasado) a eventos incorpóreos que estos cuerpos producirán (futuro). Los seres humanos pueden extraer artificialmente un solo momento de esta variación y llamarlo el presente, es decir, conseguimos hacer que el cuerpo sea un acontecimiento (Beaulieu, 2012). Podemos prestar atención a cualquier momento concedido de nuestro pasado, presente o futuro (imaginado), pero todo lo que encontramos son series de acontecimientos incorpóreos que se produjeron y pasaron a otros eventos que luego se crearon. Esta es la razón por la que el Aion "se divide hasta el infinito en pasado y futuro," (Deleuze, 2011, p. 9). Notemos que esto no es exclusivo de nuestra experiencia humana del mundo. Todas las demás entidades de igual forma simplemente captan el flujo de acontecimientos incorpóreos que se han producido a medida que lentamente dan paso a eventos adicionales que se están originando.

\footnotetext{
${ }^{10}$ Antes de continuar, es importante enfatizar que Deleuze no se adhiere a nuestra forma habitual de hablar del pasado como algo que fue presente, el presente como algo que es presente y el futuro como algo que será presente. Cuando escribe sobre el presente, (en su mayoría) se refiere a lo que sucede con los cuerpos reales, y cuando escribe sobre el pasado y el futuro, (en su mayoría) se refiere a cómo suceden los eventos incorpóreos. Con esto en mente, procedemos.
} 
A la inversa, Aión es ilimitado, mientras que Cronos es limitado y mide la acción de los cuerpos como causas y el estado de sus mezclas en profundidad; Aión es el pasado-futuro en una subdivisión infinita que esquiva siempre el presente. Cronos es "es el presente que sólo existe, y que hace del pasado y del futuro sus dos dimensiones dirigidas, de modo que se va siempre del pasado al futuro" (Deleuze, 2011, p. 61). Como el tiempo de los cuerpos mismos, Cronos es corpóreo. Si Cronos como el presente de los cuerpos divide a Aion en un pasado y futuro, se debe a que los acontecimientos incorpóreos sólo son aprehendidos o experimentados por los cuerpos, nunca por otros eventos. Por lo tanto, solo en relación con los cuerpos se puede hacer una distinción entre acontecimientos que alguna vez fueron y eventos que sucedieron luego. Sin embargo, no basta con afirmar que los cuerpos tienen su propio presente en el que persisten. Como se discutió, debe darse el caso de que los cuerpos sean producidos y alterados por los eventos incorpóreos que encuentran. Por lo tanto, Aion y Cronos deben involucrarse mutuamente.

El primer aspecto de este vínculo es que los acontecimientos incorpóreos son producidos por los cuerpos. En palabras de Deleuze, Aion es "subir a la superficie" de las "profundidades" (Deleuze, 2011, p. 98). No obstante, estos cuerpos no son entidades inmutables. Su presente es un "presente viviente variable" (p. 120), de tal manera que hay un "devenir de las profundidades" (p. 127). El presente del Cronos pasado, presente y futuro son dimensiones del tiempo, es decir, "no importa cuánto tiempo marque Cronos, siempre será suficiente o insuficiente, pero nunca demasiado o demasiado poco" (Tejeda, 2016). Esto se debe a que Cronos es la "acción de los cuerpos y la creación de cualidades corporales" (Deleuze, 2011, p. 120). Ya que los cuerpos son definidos como causas, es decir, sabemos que la acción de los cuerpos es su producción de acontecimientos incorpóreos (Faba-Zuleta, 2020). Cronos conduce al Aion: detrás de cualquier acontecimiento incorpóreo se esconde un cuerpo que lo está produciendo. La creación de cualidades corporales es la reproducción o alteración de los propios cuerpos. Como ya sabemos que los cuerpos nunca se encuentran directamente con otros cuerpos ni siquiera ellos mismos, esto debe ser en virtud de eventos incorpóreos, por tanto, el Aion también debe conducir al Cronos.

El hecho de que los cuerpos se vean afectados por eventos en términos del presente, es decir, en términos de sí mismos, apunta al hecho de que, si los eventos alteran las cualidades de un cuerpo, que depende de esas mismas cualidades. Aquí, también, los cuerpos son causas, ya que su propia naturaleza maleable determina si los eventos serán registrados, así como la medida en que tales acontecimientos causan un cambio real en un cuerpo.

Esto nos da el modelo completo del tiempo doble, es decir cualquier cuerpo atribuido registra, aprehende o experimenta una sucesión ininterrumpida 
de acontecimientos incorpóreos entrelazados. Esta sucesión es el Aion, el primer aspecto del tiempo, y es ininterrumpida precisamente, porque solamente manifiesta eventos, nunca cuerpos en sí mismos. Es una sucesión del pasado al futuro, una serie de sucesos que se han producido y que serán reemplazados por eventos que luego se producen (Deleuze, 2011). El segundo aspecto del tiempo es Cronos, que se refiere a la producción de eventos por los cuerpos, así como a la persistencia de estos cuerpos y la regeneración o alteración de sus cualidades.

Concretamente, esto significa que todo lo que experimento o cualquier cosa que un cuerpo registre es una sucesión que consiste exclusivamente en productos, en acontecimientos manifestados por cuerpos. Esta sucesión es estrictamente hablando, la producción de acontecimientos que está completamente ausente de esta época. Esto se debe a que el vínculo entre un cuerpo aislado y los acontecimientos que lo envuelven no puede hacerse presente en otro cuerpo y tampoco a sí mismo. De igual manera ocurre con la forma en que los eventos que registra afectan y posiblemente alteran un cuerpo y sus cualidades, ya que este vínculo está igualmente escondido detrás de la notable sucesión de eventos. Por lo tanto, el proceso de los cuerpos que producen acontecimientos y son reproducidos o alterados por esos eventos implica un tiempo secundario, un circuito entre cuerpos y acontecimientos que se retira detrás de las envolturas incorpóreas de las entidades. Es este modelo de tiempo el que debería incorporarse a la ontología orientada a los objetos.

\section{LOS OBJETOS Y LAS DOS FORMAS DE TIEMPO}

Para relacionar el tiempo con los objetos, primero debemos reconocer que el tiempo no es la tensión entre los objetos sensuales y sus cualidades. Incluso nuestra propia experiencia del mundo sensual siempre incluye también la asociación y disociación variable de objetos sensuales entre sí, como vimos en el ejemplo del horizonte. Además, los encuentros dinámicos entre los objetos sensuales y las cualidades no existen por sí mismos, sino sólo en relación con los objetos reales que los experimentan, aprehenden o registran. Puesto que Harman recurre regularmente a Husserl para teorizar objetos y cualidades sensuales, podemos cambiar el nombre de la tensión entre un objeto sensual y sus cualidades a un bosquejo. En este sentido, el siguiente paso es adaptar el modelo de tiempo doble de Deleuze en la estructura óntica cuádruple de Harman.

El tiempo ya no es entonces una tensión entre dos de los cuatro aspectos de los objetos. Es decir, el tiempo es doble, y cada uno de sus dos aspectos o formas implica, por un lado, "un objeto real con sus cualidades reales y, por otro, objetos sensuales con sus cualidades sensuales" (Jiménez, 2020, p. 159). Dicho de otro modo, el tiempo es una doble dinámica entre el doblete alejado de los objetos y el doblete expreso de los objetos. En este sentido, el primer aspecto del tiempo, según 
Deleuze, era el Aion, el juego variable de los acontecimientos incorpóreos y sus cualidades que es aprehendido por los cuerpos.

En la ontología orientada a los objetos, esto se convierte en el juego variable de los objetos sensuales y sus cualidades, tal y como son aprehendidos por los objetos reales. Aquí también hay un sentido en el que el mundo sensual no es el presente, sino la transición del pasado al futuro. La ontología orientada a los objetos, en este sentido, sostiene que los objetos sensuales son traducciones de los objetos reales que se esconden tras ellos (Baraglia, 2014). Lo que cualquier entidad aprehende o experimenta son, por tanto, objetos sensuales que los objetos reales han producido, y éstos sólo pueden ser sustituidos por otros objetos sensuales que los producen a continuación. Lo que permanece presente a lo largo de esta sucesión es el objeto real que registra esta variación. Como indicó Deleuze, un objeto real siempre experimenta esta sucesión en sus propios términos, es decir, "una síntesis de sucesión que remite a una sola serie" (Deleuze, 2011, p. 38), porque el hecho de que un objeto registre los acontecimientos sensuales, y cómo lo haga, depende de las cualidades reales que tenga. Esto incorpora el primer aspecto del tiempo en la ontología orientada a los objetos.

El segundo aspecto del tiempo es el Cronos, el tiempo de la producción de los acontecimientos incorpóreos por parte de los cuerpos reales, así como la reproducción o alteración de estos cuerpos al registrar los acontecimientos incorpóreos. Cronos es, en este sentido, el circuito temporal entre cuerpos y acontecimientos. Para la ontología orientada a los objetos, "es el circuito entre la mitad retirada de los objetos y la mitad sensual de los objetos" (Jiménez, 2020, p. 160). Esto se convierte en la producción de los objetos sensuales y sus cualidades por parte de los objetos reales y sus modos, así como la reproducción o alteración de los objetos reales cualificados al registrar los objetos sensuales suficientes.

Ahora bien, cambiemos el nombre por el de tiempo interno y observamos que las dos direcciones de este circuito ya se reconocen en la ontología orientada a los objetos. Por un lado, los objetos sensuales y sus cualidades son traducciones de los objetos reales que se encierran. Esto implica que los objetos sensuales son originados por los objetos reales, a partir de las cualidades reales de estos últimos. Por otro lado, vimos que el encuentro de un objeto real con los objetos sensuales puede dar lugar a un cambio actual de sus cualidades reales, ya que según Harman existen "acontecimientos relativamente raros que transforman la realidad misma de un objeto" (Harman, 2017, p. 45). De la misma manera divisamos que la apertura unilateral de los objetos reales es la condición para la posibilidad de dicho cambio.

Sin embargo, esto pone en duda la tesis de Harman de que el cambio es real, es decir, las alteraciones de las cualidades reales de un objeto son 
poco frecuentes. Parece más adecuado indicar que, mientras que el cambio real significativo es raro, el cambio real incremental podría ser bastante habitual. Si un objeto real está abierto a todos sus encuentros sensuales, entonces también debe ser posible que tal encuentro conduzca a ligeras alteraciones de las cualidades reales de un objeto, al igual que debe ser posible que simplemente conduzca a la regeneración y no a la alteración de tales cualidades. Y, por supuesto, de igual forma es totalmente posible que un encuentro sensual no tenga absolutamente ningún efecto sobre un objeto, precisamente porque un objeto real tiene cualidades reales (Jiménez, 2020). Como señala Harman, los "objetos se defienden de cualquier circunstancia a la que se enfrenten" (Harman, 2017, p. 81). Debido a las cualidades reales que tengo o, mejor dicho, que soy, puedo ser muy receptivo a una experiencia que termina cambiándome profundamente, mientras que otra experiencia puede no dejar la menor huella en mi ser.

Obsérvese también que, si bien no es necesario que un objeto real manifieste siempre una superficie sensual, puesto que puede darse el caso, simplemente, de que ningún otro objeto lo registre en ese momento, es decir, parece necesario que un objeto real registre siempre, al menos, algunos objetos sensuales si quiere persistir. Harman parece dar la razón, ya que los objetos reales son generados y regenerados por los encuentros, por consiguiente, "todo ente tiene un carácter cualitativo definido, y yo afirmaría que tener tal carácter debe significar estar articulado o construido por piezas" (Harman, 2016, p. 43). Estas piezas tienen que ser objetos sensuales. Después de todo, los objetos son la única base posible para la existencia de entidades en la ontología orientada a los objetos, y los objetos sensuales son la única clase de "objetos que pueden afectar a un objeto real" (Jiménez, 2020, p. 157). Incluso si un objeto se las arregla de alguna manera para cuidar de su propia persistencia regenerándose a sí mismo, esto tiene que desarrollarse en términos de que ese objeto real produce un objeto sensual que luego se encuentra por sí mismo.

Tomemos el ejemplo de encontrar las fuerzas en uno mismo para persistir en tiempos de dificultad. Esto no puede ser un caso en el que usted se alimente de sí mismo como un objeto real. Debe tomar la forma de que te proveas de un objeto sensual un pensamiento, un recuerdo o un sentimiento indeterminado que te permita persistir. También debemos señalar que esto no quebranta los objetos reales en los objetos sensuales que los regeneran, porque existe una diferencia “de tipo entre las dos dimensiones de los objetos" (Jiménez, 2020, p. 158). Si la generación de objetos sensuales por parte de los objetos reales es una cuestión de traducción, lo mismo ocurre con la incorporación de la fuerza o el impacto de los encuentros sensuales al objeto real. 
Como se ha dicho anteriormente, esta doble teoría del tiempo para la ontología orientada a los objetos implica que los objetos participan en tres síntesis temporales (Harman, 2017). Cada síntesis implica tanto el tiempo manifiesto como el tiempo interno. En primer lugar, los objetos reales producen objetos sensuales cualificados que se contraen en la sucesión ininterrumpida de las experiencias o aprehensiones de otros objetos reales. Recordemos el ejemplo de los objetos sensuales que se añaden gradualmente a la experiencia de un observador de una línea de horizonte. En segundo lugar, los objetos reales y sus cualidades se mantienen y posiblemente se alteran ya sea ligera o significativamente por la sucesión de objetos sensuales que encuentran.

En tercer lugar, siempre que un objeto real forja una relación con un objeto sensual, esto implica inmediatamente la generación de un nuevo objeto real. Como dice Harman, "toda relación genuina forma un nuevo objeto" (Harman, 2015, p. 43). Como sugiere la ontología orientada a los objetos, cuando percibo un atardecer, entablo una conversación con mi pareja o pienso en mi ciudad natal, la percepción, la conversación y el pensamiento no son sólo relaciones. Son entidades reales en toda regla que no pueden reducirse ni a mí ni a los objetos sensuales del otro lado. Estos nuevos objetos también están inmediatamente inmersos en el tiempo doble, y esto constituye el último ingrediente de la dinámica temporal de la realidad. Los objetos sintetizan el mundo sensual, los objetos se sintetizan en la medida en que el mundo sensual les afecta, "y los objetos sintetizan nuevos objetos en la medida en que se relacionan con el mundo sensual" (ibíd.).

Obsérvese que el desarrollo de la ontología orientada a los objetos con un tiempo doble evidente y un tiempo interno deja completamente intacta la cuádruple estructura de los objetos de Harman. La cuestión es ahora si esta teoría del tiempo doble es una respuesta adecuada a las críticas de Gratton y Wolfendale. La primera es que debe dar cuenta de la producción, alteración y aniquilación de los objetos reales indicados. Hemos notado que el tiempo interno es, en efecto, la condición de posibilidad de tales acontecimientos. El segundo punto se refiere a la temporalidad de los diez vínculos entre los aspectos del cuádruple objeto. Dado que el efecto combinado del tiempo interno y el tiempo visible incluye todos los aspectos del cuádruple objeto, esto también se explica ahora. Por último, la teoría del tiempo de la cuádruple dimensión tenía que dar cuenta de la persistencia de los objetos sin plantear un tiempo que existiera por encima de ellos. Esto también se explica en la teoría del tiempo doble. Los objetos persisten o perecen en la medida en que son no regenerados por los objetos sensuales que atrapan, es decir, sobre los que se apoyan, y esto se justifica por la apertura unilateral de los objetos reales en relación con su tiempo interno (Ramírez, 2017). 


\section{CONCLUSIÓN}

Al adoptar la teoría del tiempo doble de Deleuze en la ontología orientada a los objetos de Harman aumentará la coherencia y consistencia. El hecho de que la primera pueda injertarse en la segunda también puede llevarnos a reconsiderar la distancia relativa entre las filosofías de Harman y Deleuze. Harman ha argumentado que, si los metafísicos continentales se dividieran nítidamente en escuelas de pensamiento específicas, él y "Deleuze se situarían en otras diferentes" (Harman, 2014, p. 237). Harman da varias razones para ello, pero lo que nos interesa aquí es que una de esas razones son sus nociones aparentemente incompatibles de tiempo y devenir. En la ontología orientada a los objetos, los objetos son los constituyentes básicos de la realidad. Si es el caso que nada excepto otros objetos preceden a los objetos, entonces todo el tiempo debe ser por definición una dinámica entre entidades bien formadas.

Por otro lado, Harman sostiene que un rasgo definitorio de los filósofos del devenir, como Deleuze, es que sitúan todo el tiempo antes de los objetos bien formados. Tales filósofos sostendrían que "las entidades son sólo afloramientos derivados de algún devenir preindividual más profundo" (Harman, 2014, p. 235). Por debajo o más allá de nuestro mundo de objetos discontinuos, postulan una continuidad de procesos dinámicos, así como un tiempo continuo. Harman señala: "Un filósofo del devenir es aquel que niega que el mundo se entienda mejor en términos de cosas individuales o instantes individuales de tiempo (...) y el tiempo es una duración continua más que una serie de fotogramas cinematográficos aislados" (Harman, 2014, p. 239).

Desde este punto de vista, el tiempo real siempre ha sucedido cuando se llega a las entidades reales y discretas, porque el tiempo pertenece a la continuidad de los procesos preindividuales que dan lugar a dichas entidades. Por lo tanto, las filosofías del devenir sitúan los objetos bien formados al final del tiempo, ya que el tiempo pertenece exclusivamente a todo lo que ocurre antes de que una entidad real se exprese en el mundo. Harman se encuentra aquí de acuerdo con la conocida lectura de Peter Hallward de la metafísica del reino virtual de Deleuze, es decir, "dado que sólo reconoce una relación unilateral entre lo virtual y lo real, no hay lugar en la filosofía de Deleuze para ninguna noción de cambio, tiempo o historia que esté mediada por la actualidad" (Hallward, 2006, p. 162). Las nociones de un reino virtual y de un tiempo que precede completamente a los individuos son obviamente anatema para la ontología orientada a los objetos. Sin embargo, al mismo tiempo, hemos visto que una teoría deleuziana del tiempo es conmensurable con una ontología orientada al objeto, y que esta última incluso gana en consistencia si incorpora la primera. 
¿Cómo podría ser esto? La respuesta obvia es que la ontología orientada a los objetos de Harman es efectivamente inconmensurable con la ontología de Deleuze, pero esta inconmensurabilidad concierne principalmente a la ontología que encontramos en Diferencia y Repetición.

Para nuestro caso sería arcaico tal y como se teoriza en Diferencia y repetición usar esa ontología con la de Harman. Además, vimos que en Lógica del Sentido se basa en una noción de cuerpos como sustancias y causas "con sus tensiones, sus cualidades, sus relaciones" (Deleuze, 2011, p. 9) que se relegan a sí mismos, lo que significa que estos cuerpos están ontológicamente enmascarados por "revestimientos superficiales" (Deleuze, 2011, p. 194) o acontecimientos superficiales. Y lejos de abarcar un tiempo único y continuo en una profundidad virtual, esta ontología de cuerpos y superficies implicaba que "el tiempo debe ser captado dos veces, de dos maneras complementarias, aunque mutuamente excluyentes" (Hallward, 2006, p. 133). Este es el punto en el que empiezan a surgir las compatibilidades entre la ontología orientada a los objetos y la ontología deleuziana. En este sentido, cómo llamaríamos al creciente interés sobre los cuerpos, las máquinas y los ensamblajes en las obras de Deleuze después de Diferencia y Repetición, sino es por una creciente orientación hacia los objetos A la luz del análisis anterior, la cuestión puede ser muy clara si las filosofías de Deleuze y Harman se superponen o no, sino más bien cuánto lo hacen realmente.

Para terminar una última observación, sobre las numerosas críticas a la ontología orientada a los objetos que se han mencionado al principio de este trabajo. En este sentido, es posible abordar y remediar el problema del tiempo en la ontología orientada a los objetos, y si esto proporciona una respuesta adecuada a lo que Gratton y Wolfendale consideran el peor problema que actualmente acecha a este modelo de ontología, entonces es lógico que también se puedan remediar otros defectos menos condenatorios de esta teoría. Al menos desde el punto de vista de que la noción de realidad podría ser una multitud de entidades maleables que no pueden reducirse a una capa, sustancia o estructura privilegiada, ni disolverse en el conjunto de sus operaciones, en este sentido, sigue siendo lo suficientemente fresca, convincente y, sobre todo, inquietante como para justificar un análisis más profundo. 


\section{BIBLIOGRAFÍA}

Beaulieu, Alain (2012). Cuerpo y Acontecimiento. La estética de Gilles Deleuze. Buenos Aires: Letra Viva.

Baraglia, Rodrigo. (2014). "Sobre la ontología orientada a objetos Una introducción a la filosofía de Graham Harman”. Revista Luthor, 20, pp. 25-37.

Casado, G. (2019). "Reflexión crítica sobre el brutalismo". Arquitectura y Urbanismo, $\mathrm{XV}(2), 5-20$.

Deleuze, Gilles. (1995). Proust y los signos, trad. Francisco Monge, Barcelona: Anagrama.

Deleuze y Guattari. (1997). ¿Qué es la filosofía? Barcelona: Anagrama.

Deleuze, G. (2002). "Últimos textos: El 'Yo me acuerdo'. La inmanencia: una vida ...".

Contrastes. Revista interdisciplinar de filosofía, 7, 2002, pp. 219-237.

Deleuze, Gilles. (2006). Diferencia y repetición. Buenos Aires: Amorrortu.

Deleuze, Gilles. (2008). Dos regímenes de locos. Barcelona: Pre-Textos.

Deleuze, Gilles. (2011). Lógica del sentido. Madrid: Paidós.

Faba-Zuleta, Paulina. (2020). "El cuerpo como acontecimiento. Las formas de operar de lo político en el arte de Ana Mendieta”. Arte, Individuo y Sociedad 321, pp.133-54.

Gratton, Peter. (2014). Speculative Realism. Problems and Prospects. London: Bloomsbury.

Hallward, Peter. (2006). Out of This World-Deleuze and the Philosophy of Creation.

London: Verso.

Harman, Graham. (2005). Guerilla Metaphysics: Phenomenology and the Carpentry of Things. Chicago: Open Court.

Harman, Graham. (2015). Hacia el realismo especulativo. Ciudad Autónoma de Buenos Aires: Caja Negra.

Harman, Graham. (2014) "Whitehead and Schools X, Y, and Z." The Lure of Whitehead, Nicholas Gaskill and A.J. Nocek (eds.), pp. 231-248. Minneapolis: University of Minnesota Press.

Harman, Graham, (2016). El objeto cuádruple. Una Metafísica de las cosas después de Heidegger. Barcelona: Anthropos.

Harman, Graham. (2017). Immaterialism: Objects and Social Theory. Cambridge: Polity.

León, Eduardo Alberto. (2020). “Deleuze y Žižek. Una visión de Paralaje”. Thémata. Revista De Filosofia, 61, pp. 11-28.

Jiménez, Luis Alberto. (2020). Reseña de "Ontología Orientada a Objetos una nueva teoría del todo”. Análisis. Revista de investigación filosófica, 7, 1, pp. 151-155. 
Morton, Timothy. (2020). Magia realista: objetos, ontología y causalidad. London: Open Humanities Press: http://openhumanitiespress.org/books/titles/magia-realista

Ontología orientada a objetos - HiSoUR Arte Cultura Historia. https: (www.hisour.com) Consultado: 27-01-2021.

Ramírez, Mario Teodoro. (2017). “Ontología de la resistencia”. Valenciana, 10, 19, pp. 7-28.

Tejeda, Javier. (2016). “Las dos caras del tiempo: Aion y Cronos”. Revista Cultural: Le Miau Noir. https:/www.lemiaunoir.com/tiempo-aion-cronos/

Wolfendale, Peter (2014). Object-Oriented Philosophy - The Noumenon's New Clothes. Falmouth: Urbanomic. 